\title{
ИССЛЕДОВАНИЕ ВОПРОСОВ СОЗДАНИЯ РАСКЛАДКИ В АВТОМАТИЧЕСКОМ РЕЖИМЕ НА ОСНОВЕ АНАЛИЗА ХАРАКТЕРИСТИК ДЕТАЛЕЙ
}

\section{INVESTIGATION OF THE ISSUES OF CREATING A LAYOUT IN AUTOMATIC MODE BASED ON THE ANALYSIS OF THE CHARACTERISTICS OF PARTS}

\section{A. Murtazina V. Mironov A. Gusev}

Summary. The objective of nesting is the problem of optimizing cutting, which consists in finding the best scheme for a set of different parts on natural leather or material. The high quality requirements for the products, combined with the heterogeneity of the material, fulfill a difficult practical solution. There are very few results in the literature on the issue of placement of parts, especially on leather. Moreover, most approaches impose some additional constraints on the layout scheme specific to the particular application in question. This article discusses the method of nesting on the geometric characteristics of parts.

Keywords: lay out, industry, part contour, the radius of curvature, pattern recognition, optimization nesting of blanks.
Муртазина Альфия Рустямовна

К.т.н., дочент, ФГБОУ ВО «Российский государственный университет имени А.Н. Косыгина (Технологии. Дизайн. Искусство)», Москва aly1029@yandex.ru

Миронов Владислав Петрович К.т.н., дочент, ФГБОУ ВО «Российский государственный университет имени А.Н. Косыгина (Технологии. Дизайн. Искусство)», Москва mironov-vp@rguk.ru

Гусев Александр Олегович

Аспирант, ФГБОУ ВО «Российский государственный университет имени А.Н. Косыгина (Технологии. Дизайн. Искусство)», Москва alex@gusev.xyz

Аннотация. Задача раскладки — это проблема оптимизации раскроя, которая заключается в нахождении наилучшей схемы расположения для набора разных деталей на натуральной коже или материале. Высокие требования к качеству, предъявляемые к продуктам, в сочетании с неоднородностью материалов делают задачу сложной для практического решения. В литературе очень мало результатов по проблеме размещения деталей, особенно на коже. Более того, большинство подходов налагают некоторые дополнительные ограничения на схему раскладки, относящиеся к конкретному рассматриваемому приложению. В этой статье рассматривается разработка метода раскладки на основе геометрических характеристик деталей.

Ключевые слова: размещения деталей, промышленность, контур детали, радиус кривизны, распознавание образов, оптимизации раскроя.

материалов и затраты, связанные с погрузочно-разгрузочными работами.

Программное обеспечение для раскроя с раскладкой деталей должно минимизировать использование материала и межлекальные отходы при выполнении операции резания, обеспечивая использование материала на 80-90\%. Однако подобная задача достаточно сложная: необходимо учитывать рисунок на материале, его толщину и принимать во внимание ориентацию детали, её геометрические особенности. Необходимо отметить, что производители должны поставлять качественные детали по мере необходимости, чтобы соответствовать внутреннему производственному процессу и внешним требованиям, то есть графику поставки клиенту. Таким образом, при построении раскладки в автоматическом 


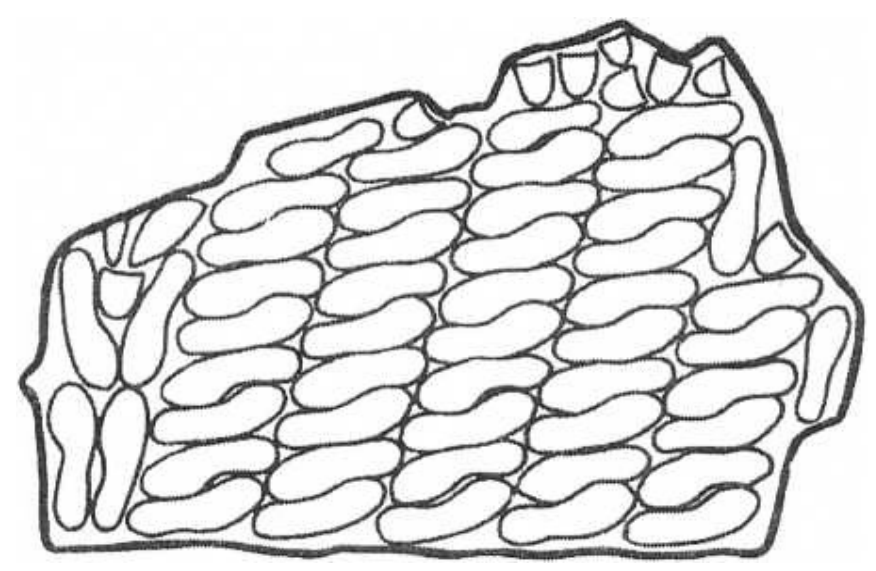

Рис. 1. Раскладка деталей обуви

режиме необходимо решить несколько задач: определение качества сырья (обнаружение дефектов на материале); распознавание и классификация деталей для их правильного расположения; определить наличие рисунка/ узора на материале и предложить оптимальный вариант совмещения.

Первую и вторую задачи возможно решить с помощью нейросетей [1] и библиотек обработки изображений, например OpenCV, позволяющей выполнить предварительную обработку изображения и подготовить его для последующих этапов распознавания и передачи информации в нейронную сеть. В качестве описания контуров деталей можно использовать рекуррентный компьютер [2] для регулирования количества точек. Этап построения раскладки слабо автоматизирован для деталей сложной формы [3,4], а также описанных с помощью сплайнов или кривых. В данной статье проводились исследования по автоматизации раскладки на деталях обуви.

На рис. 1. показан результат процесса раскладки деталей обуви. Если соединить точки касания деталей (с учетом технологического промежутка между ними), то на раскладке можно увидеть некоторую закономерность. Эти закономерности явно зависят от геометрии области и деталей. Следовательно, необходимо тщательно изучить конфигурацию областей и деталей для выявления особенностей, которые способствуют нахождению алгоритма при построении раскладок. В дальнейшем появится возможность соединять в блоки детали определенного класса.

В работе рассматривается плоская деталь, представленная последовательностью точек, которые образуют границу детали. Внутренность детали считается окрашенной в белый цвет, без текстуры, так как точки получены после операции бинаризации. Но если бы изобра- жение было с текстурой, например, теснением на коже, то можно было бы говорить о характерных признаках деталей, цветности пикселей, предлагавшиеся в литературе [5], где применялись общие методы выделения характерных параметров текстур объектов таких, как энергия, энтропия, контраст, однородность, корреляция и другие.

Проведённые эксперименты показали, что не все признаки оказываются полезными и актуальными для рассмотрения и приведения к функциям для дальнейших расчетов параметров плоского объекта, так как зависят от координат и являются неканоническими. Поэтому решено построить процедуры для вычисления основных параметров плоского объекта - площади и периметра, а также заинтересовавшей характеристики - числа вогнутостей детали, которая является топологической, носит интегральный характер, определяет форму детали и может служить для решения задач кривых определенного класса, в частности классификации, раскроя, аппроксимации.

Следующей частью работы после вычисления площади и периметра плоского объекта является изучение локальных характеристик таких, как кривизна деталей контура, некоторые элементы аппроксимационного плана. Эти характеристики не интегральные, изменяются по координатам. Они в свою очередь могут использоваться для анализа динамики режущих инструментов, задач аппроксимации и других. Исходная информация для решения данных задач - последовательность координат плоского объекта, полученная после процесса скелетизации. Возможны разные направления обхода контура.

$$
\begin{aligned}
& \text { Периметр } \\
& P=\sum_{i=1}^{n-1} \sqrt{\left(x_{i+1}-x_{i}\right)^{2}+\left(y_{i+1}-y_{i}\right)^{2}}+ \\
& +\sqrt{\left(x_{1}-x_{n}\right)^{2}+\left(y_{1}-y_{n}\right)^{2}}
\end{aligned}
$$




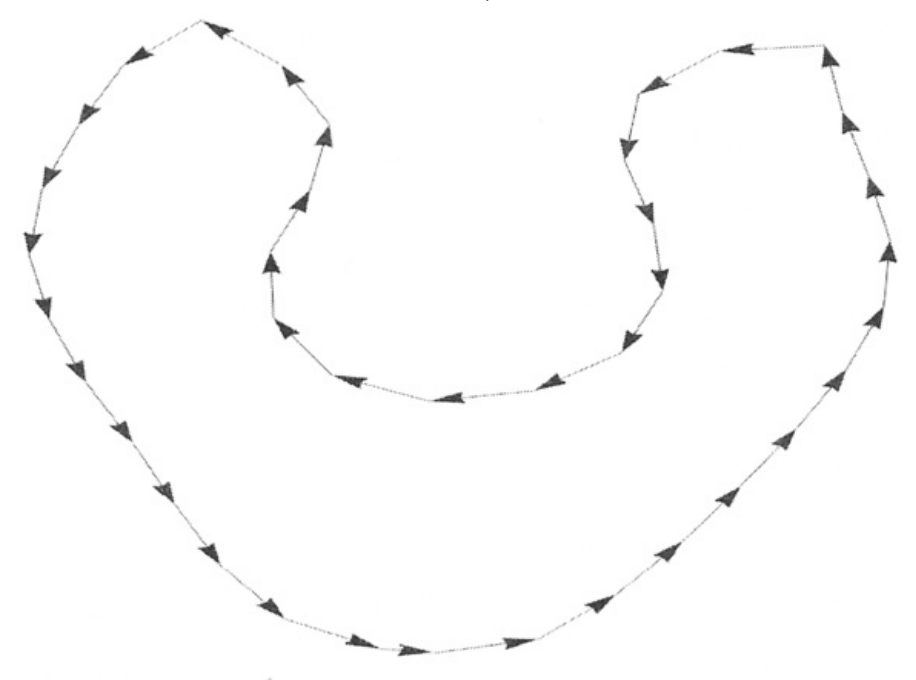

Рис. 2. Направление обхода контура

где $x_{i}, x_{n}$ - координаты векторов по оси $\mathrm{X}, y_{i}, y_{n}-$ координаты векторов по оси Y.

\section{$\sqcap \wedge$}

$$
\begin{aligned}
& S=\frac{1}{2} \sum_{i=1}^{n-1}\left(x_{i} * y_{i+1}-y_{i} * x_{i+1}\right)+ \\
& +\frac{1}{2}\left(x_{n} * y_{1}-y_{n} * x_{1}\right)
\end{aligned}
$$

\section{Наличие выпуклостей/вогнутостей}

Эта характеристика определяется следующим образом: совершается обход в определенном направлении (положительном, против часовой стрелки, или отрицательном, по часовой). В работе приняли положительное направление и формировали вектор направления движения в пошаговом режиме. При сравнении изменения направления (положительное, отрицательное) этих последовательных векторов относительно друг друга (рис. 2), сформировалась последовательность знаков изменений и направлений.

Для получения последовательности знаков изменений, которые и будут показывать, есть ли у детали вогнутость, была найдена разность координат по $X$ и по У векторов контура, после чего полученные разности записаны в 2 столбика и перемножены крест - накрест, и снова вычислена их разность.

\section{Рахиус кривизны}

Чтобы вычислить кривизну каждого участка контура, рассчитываются параметры участков - косинусы и си- нусы углов между векторами контура. Косинус угла вычисляем по формуле:

$$
\begin{aligned}
& \frac{\left(a_{1} * a_{2}+b_{1} * b_{2}\right)}{|a| *|b|} \\
& \text { где } a_{1}=\left(x_{1}-x_{2}\right), \text { а } a_{i}=\left(x_{i}-x_{i+1}\right) \\
& b_{1}=\left(y_{1}-y_{2}\right), \text { a } b_{i}=\left(y_{i}-y_{i+1}\right) \\
& |a|=\sqrt{a_{1}^{2}+a_{2}^{2}},|b|=\sqrt{b_{1}^{2}+b_{2}^{2}}
\end{aligned}
$$

Поскольку знаменатель дроби всегда будет положительным, то можно рассматривать только числитель дроби, для которого необходимо определить знак, указывающий на расположение искомого угла в координатной четверти. Синус рассчитывается из отношения векторного произведения векторов контура к длине этих векторов. Далее вычисляем арксинус полученного значения и отслеживаем знак косинуса: если он больше нуля, то из числа п вычитаем полученное значение арксинуса; иначе значение остается. На заключительном этапе значение делим на длину вектора, у которого определяем угол поворота:

$$
\frac{\Delta ц}{\Delta s^{\prime}}
$$

$\Delta ц$ - изменение угла между векторами, $\Delta s-$ расстояние между началами векторов. В результате вычислений получаем оценку

$$
\frac{1}{R^{\prime}}
$$

где $R$ - радиус кривизны, приближенное значение которого можно также получить из описанной окружности вокруг треугольника, образованного векторами. 


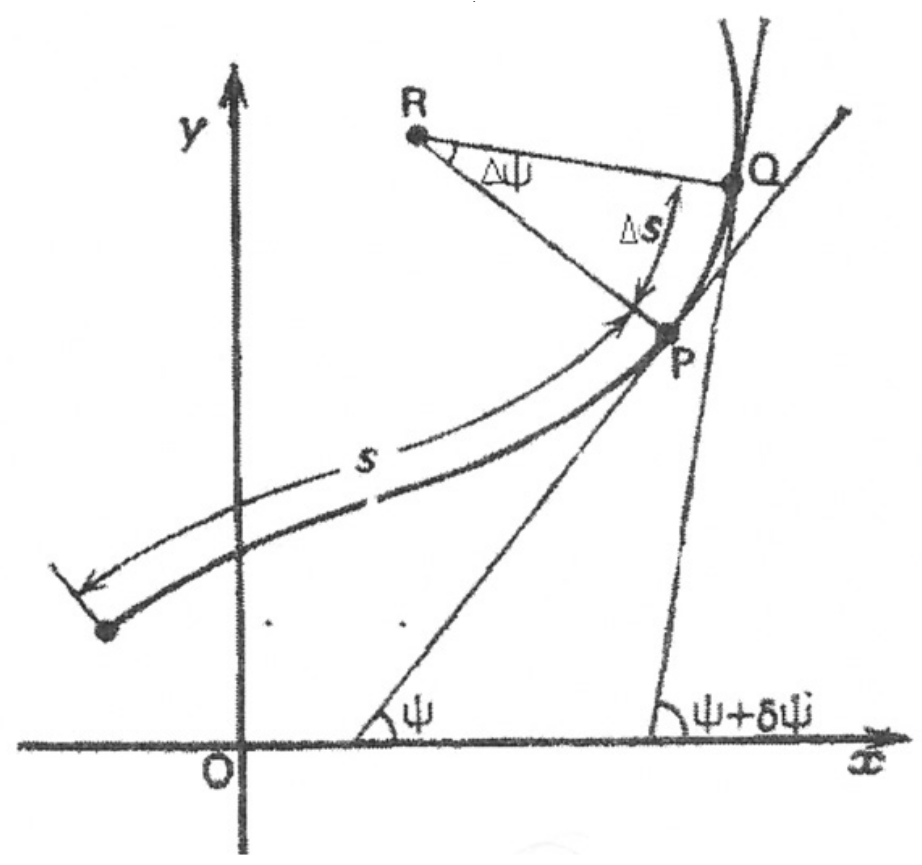

Рис. 3. Часть контура детали

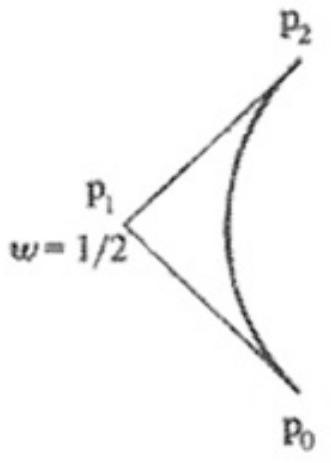

a

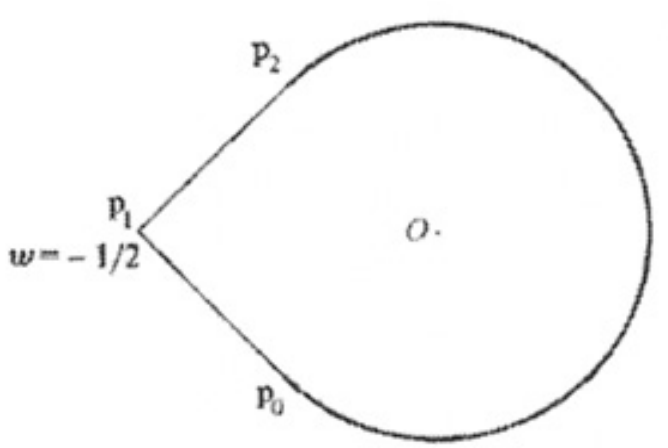

6

Рис. 4. Вес точки $p_{l}$ : а) положительный; б) отрицательный [6]

\section{РаАиусы вписанных и описанных окрУжностей}

Использовав формулу линейно-круговой аппроксимации [6], можно построить приближение дуги окружности:

$$
\begin{aligned}
& r(t)=\frac{(1-t)^{2} p_{0}+2 t(1-t) щ p_{1}+t^{2} p_{2}}{(1-t)^{2}+2 t(1-t) щ+t^{2}}, 0 \leq t \leq 1 \\
& \left|p_{0}-p_{1}\right|=\left|p_{2}-p_{1}\right|=p \operatorname{tg}(\sigma / 2), \\
& \left(p_{0}-p_{1}\right) \cdot\left(p_{2}-p_{1}\right)=\left|p_{0}-p_{1}\right|\left|p_{2}-p_{1}\right| \mid \cos (\text { б }) \mid
\end{aligned}
$$

$$
\cos (\sigma / 2)=щ
$$

Здесь щ - вес средней точки, который может быть как положительным, так и отрицательным (рис. 4).

Анализируя треугольник, полученный последовательностью 2 векторов на 3 точках, можно вычислить радиус-вектор центра вписанной окружности, по которому определить радиус окружности и точки касания. Нахождение точек касания позволят определить угол 6. Как показывают расчеты, значение $\cos 6$ дает вес средней точки при рациональной аппроксимации. Значение этих весов позволяет строить кусочно-линейную ап- 
проксимацию, что может понадобиться при дальнейшем рассмотрении детали.

\section{А^горитм совмешения контуров}

Функция Cross применяем для поиска периметра, площади и числа вогнутостей. Последовательность знаков из массива значений Cross, определяющих вогнутости, образуют шум. Для его устранения создается массив Signs, который заполняется значением, равным количеству подряд идущих элементов Cross с одинаковым знаком. При этом это количество имеет тот же знак, что и последовательность чисел в массиве Cross. Так, например, массив Signs может содержать подряд идущие числа 5,-2,4 - это значит, что в контуре сначала идут 5 пар линий соответствующие выпуклости, затем две пары линии соответствующие вогнутости, и далее 4 пары снова выпуклость. Если на пути обхода массива Cross попадаются нули (прямые линии), то и в массив Signs просто добавляется 0, столько же раз, сколько он встречается в массиве Cross. После формирования Signs выполняется алгоритм просеивания этого массива, при котором ищется элемент, абсолютное значение которого меньше или равно значению сглаживания, подаваемое на вход функции. Значения найденных элементов меняют знак и складываются с предыдущим, а оригинальный элемент удаляется. После массив просматривается на существование пар значений, стоящих рядом и имеющих один знак. Такие пары складываются, результат их суммы записывается вместо одного элемента, а второй просто удаляется из массива. С нулем никаких действий не происходит. Например, при последовательности 5,- 2,4,0,0 и сглаживании 2, после первой обработки получится результат 7,4,0,0, а после второй - 11,0,0. Далее в цикле отбираются пары рядом расположенных линий, которые замыкаются в треугольник, вычисляются радиусы вписанной и описанной окружности, затем радиус кривизны. После полученная информация заполняется массив о контуре детали.

Для реализации совмещения контуров необходимо создать вспомогательный класс (Comperelnfo), хранящий следующую информацию: индексы начальной и конечной точки первого и второго контуров; длину совмещаемой части контура; среднюю сумму значений, определяющих выпуклость; среднюю разницу значений кривизны; максимальную и минимальную сумму значений, определяющих выпуклость; максимальную и минимальную разницу значений кривизны; рейтинг совмещения.

Алгоритм о выбранных для совмещения контурах можно описать следующим образом:

1. Установить точку поиска совмещения первого и второго контура в ноль.

2. До тех пор, пока знак, определяющий выпуклость \} вогнутость не изменится, смещаться на точку вперед, вычисляя сумму разностей кривизны, а также сумму сумм, определяющих выпуклость। вогнутость, на всем участке.

3. Поделить вычисленные значения на длину пройденного участка, получив среднее арифметическое этих значений. Записать все вычисления в экземпляр класса Comperelnfo и добавить его в массив контуров.

4. Изменить точку поиска совмещения второго контура на ту, где остановилась предыдущая попытка совмещения.

5. До тех пор, пока точка поиска совмещения второго контура не будет равна последней точке, возвращаться в пункт 2.

6. Поставить точку поиска совмещения второго контура в ноль, а точку поиска совмещения первого контура переместить вперед, в место, где изменяется знак, определяющий выпуклость\вогнутость.

7. До тех пор, пока точка поиска совмещения первого контура не будет равна последней точке, возвращаться в пункт 2.

Таким образом получается многократный обход второго контура вокруг отдельных отрезков первого контура. После прохождения алгоритма составляется рейтинг. Для него создаются отдельные переменные, в которые помещаются максимальные и минимальные значения сумм, определяющих выпуклость\вогнутость, разниц кривизны, а также длины совмещенных участков. Затем, общее минимальное значение на всем контуре каждой из этих величин делится на соответствующее значение каждого участка. Таким образом получается 6 величин сравнения всех параметров совмещения, которые нормированы от 0 до 1. 7-й величиной является длина участка, деленная на общую максимальную длину участка, найденную при обходе контура. Так как рейтинг должен быть распределен от 0 до 100, все эти величины необходимо суммировать, предварительно умножив на определенный коэффициент. Экспериментально было выявлено, что длина участка должна иметь большую силу при составлении рейтинга, так как массив совмещенных участков зачастую имеет довольно большое количество «идеальных» по всем параметрам отрезков, но имеющих длину 1 или 2 линии, что, конечно же, для совмещения совсем не подходит. Чтобы избежать появления таких «артефактов» вычислений, длине участка был выставлен коэффициент 28, а всем другим равный коэффициент 12. Итого получается $12 * 6+28=100$.

\section{Результаты}

\section{(Results) и Обсуждение (Discussions)}

В идеале для гладкой прямой количество изменений знаков при циклическом рассмотрении показывает число 


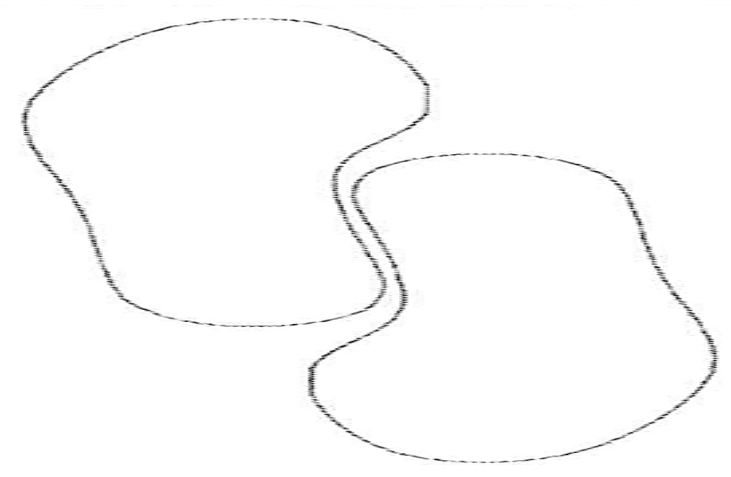

Рис. 5. Совмещение деталей

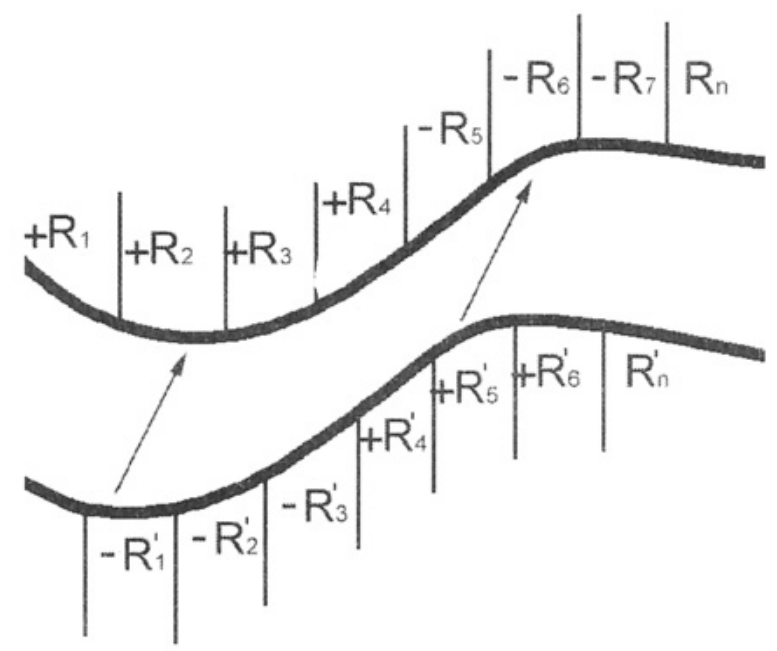

Рис. 6. Фрагмент совмещения 2 контуров.

вогнутостей детали. На практике дискретность представления приводит к тому, что нужна операция сглаживания. Для этой операции экспериментально подбирается коэффициент сглаживания, чтобы удалить близко расположенные точки контура детали, избавится от шума и в то же время не исказить деталь настолько, что нельзя будет понять, какая изначально конфигурация была у плоского объекта. Кроме этого, полученную последовательность следует подвергать фильтрации. Для отбрасывания небольших изменений знаков, связанных с дискретностью представления. Последовательность знаков разбиваем на блоки, длина которых будет равна заданному коэффициенту фильтрации. В полученных блоках сравниваем значение функции Sign первого и последнего элемента, и если они одинаковые, то все элементы противоположного знака между ними меняют свой знак.

Так как обход контура совершался в положительном направлении, то есть против часовой стрелки, положительный знак у значений кривизны наблюдается в области предположительной выпуклости фигуры, а отрица- тельный знак - в области вогнутости. Если суммировать значения кривизны на участках двух контуров фигур и наложить условие $\left|R_{i}+R_{i}^{\prime}\right|<\mathrm{e}(\mathrm{e}-$ фиксированное малое значение), можно говорить о совмещении деталей и соединении их в блоки для построения раскладки (рис. 6).

Исследование кривизны контура объекта показывает, что оценивается радиус кривизны, который определяется как радиус описанной окружности. Радиус кривизны связан с процесса формирования плоских объектов, что позволяет совмещать контуры деталей и соединении их в блоки для построения раскладки.

Учитывая, что часто в программном обеспечении файл CSV содержит необходимые для предложенного алгоритма данные о деталях, который загружается непосредственно в механизм размещения, то его можно использовать для создания функции автоматического размещения, что позволит сэкономить временя, сократить отходы и эффективно использовать материал. 


\section{ЛИТЕРАТУРА}

1. Григорян М.П., Миронов В.П. Выявление дефектов текстуры при компьютерном сканировании кожи. В сборнике: Инновационное развитие легкой и текстильной промышленности. Сборник материалов Всероссийской научной студенческой конференции. 2017. С. 27-30.

2. Муртазина А.Р., Миронов В.П., Разин И.Б. Алгоритм сжатия данных. Дизайн и технологии. 2014. № 43 (85). С. $51-55$.

3. Sheng Quan Xie, Gary Wang, Y. Liu. Nesting of two-dimensional irregular parts: An integrated approach. International Journal of Computer Integrated Manufacturing 20(8):741-756. December 2007.

4. K. Ramesh, N. Baskar. The Simple Genetic Algorithm Approach for Optimization of Nesting of Sheet Metal Parts in Blanking Operation. Journal of Advanced Manufacturing SystemsVol. 14, №. 01, pp. 41-53 (2015).

5. Шапиро Л., Стокман Д. Компьютерное зрение - М.: Бином. Лаборатория знаний, 2013.761 с.

6. Голованов Н.Н. Геометрическое моделирование: учебник для учреждений высш. проф. образования - М.: Издательский центр «Академия», 2011. 272 с.

7. Фокс А., Пратт М. Вычислительная геометрия. Применение в проектировании и на производстве /пер. с англ.— М.: Мир, 2006. 304 с.

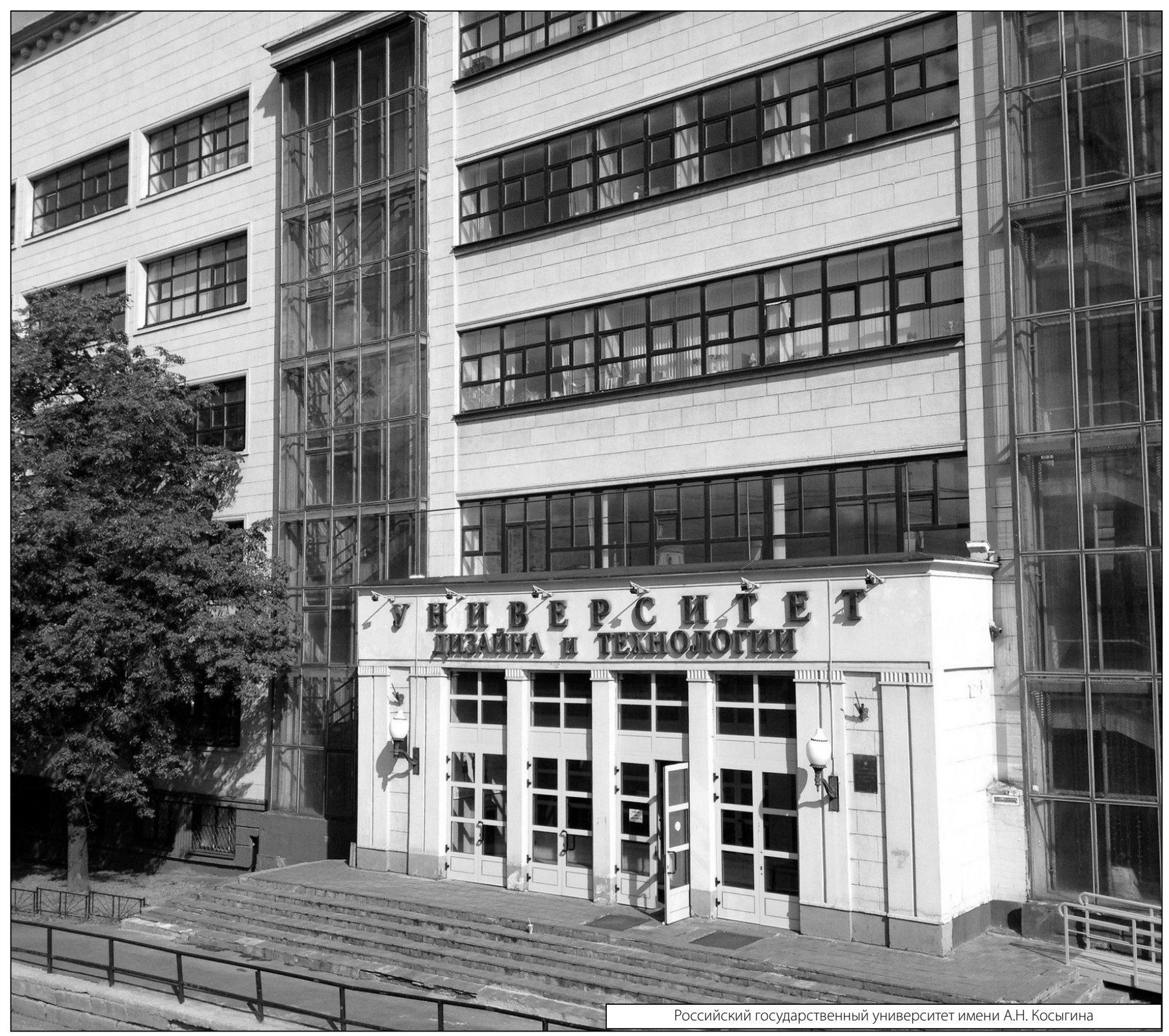

